\title{
Exclusion of long heterologous insertions and deletions from the pairing synapsis in pneumococcal transformation
}

\author{
Franck Pasta and Michel A. Sicard
}

Laboratoire de Microbiologie et de Génétique Moléculaire du CNRS, Université Paul Sabatier, 118 Route de Narbonne, 31062 Toulouse Cedex, France

\author{
Author for correspondence: Franck Pasta. Tel: +3361 3359 71. Fax: +33 61335886. \\ e-mail: pasta@ibcg.biotoul.fr
}

\begin{abstract}
We have studied the mode of recombination of six insertions during genetic transformation of Streptococcus pneumoniae. The six heterologous insertions are located at the same site in the ami locus of the pneumococcal chromosome; insertion sizes range from 4 to $1374 \mathrm{bp}$. With respect to singlepoint markers we found that the number of transformants in one-point crosses is reduced, while the number of wild-type transformants in two-point crosses is drastically increased, what we call hyper-recombination. The magnitude of the shift is correlated with the size of the insert. This effect could result either from a special repair pathway of multibase heteroduplexes or from the exclusion of multibase heterologous insertions out of the pairing synapsis. To test these hypotheses we have used insertions in two kinds of three-point crosses. The repair model predicts that the excess of wild-type transformants remains in one set of crosses but is suppressed in the second set. The results we obtained are reversed, ruling out the hypothesis of a repair process, but in agreement with predictions based on the exclusion model. Moreover, we have re-examined the situation of deletions, our previous results suggesting that deletions were likely to be converted at the heteroduplex step. Genetic evidence we obtained in this work no longer supports this hypothesis. Thus, long heterologous insertions are partly excluded at the pairing step.
\end{abstract}

Keywords: Streptococcus pneumoniae, recombination, insertions, deletions, heteroduplexes

\section{INTRODUCTION}

During pneumococcal transformation, one DNA strand enters the cell and recombines with the chromosome thereby inducing the formation of a donor/recipient heteroduplex joint (for a review, see Lacks, 1988). Once replicated, such heterozygotes generate one transformant and one recipient genotype. However, mismatches may undergo repair prior to replication. The origin of this hypothesis was the discovery that several mutations are poorly transformable in wild-type Streptococcus pneumoniae strains (Ephrussi-Taylor \& Gray, 1966). Subsequently, mutants were found, called bex, which integrate all markers with the same high efficiency (Lacks, 1970; Tiraby \& Sicard, 1973a). Further studies showed that donor/recipient mismatches may trigger a complete excision of the donor DNA from the heteroduplex structure, followed by a resynthesis of the recipient strand

Abbreviations: ami-ins, ami-insertion sequence; RF, replicative form.
(Méjean \& Claverys, 1984). This mismatch repair system, which is absent in bex mutants, has been called Hex. The mutator phenotype of hex strains strongly suggests that it also acts on mismatches spontaneously arising in DNA (Tiraby \& Sicard, 1973b; Tiraby \& Fox, 1973). Heteroduplexes $A / G$ and $C / T$ corresponding to transition mutations are efficiently corrected by Hex. Those resulting from transversions are partly repaired, except $\mathrm{C} / \mathrm{C}$ and A/G (for a review, see Claverys \& Lacks, 1986). Mispairs corresponding to small heterologous insertions, i.e from 1 to 4 bases, are also processed by Hex (Gasc et al., 1987). Due to the multiplicity of mispairs processed, Hex is considered as a generalized mismatch repair system.

Heteroduplexes corresponding to heterologous insertions longer than 4 bases escape the action of Hex (Gasc et al., 1987). Moreover several studies showed that long deletions do not behave as point mutations during transformation. Ghei \& Lacks (1967) found that the kinetics of integration were much slower for deletions than for point mutations. Also, the transformation efficiency of deletions is reduced (Lacks, 1966; Lefevre et al., 1989). Moreover, 
in two-point crosses, when donor deletions are used to transform a strain containing a linked point mutation, wild-type recombinants occur in very large excess relative to the physical distance separating the two mutations (Lefevre et al., 1989). This hyper-recombination could have two origins. First, the heteroduplex forms a multibase loop which might be corrected to the wild-type. Second, recombination might frequently stop at the region of heterology, excluding it from the pairing synapsis. Genetic evidence that we previously obtained for long deletions argues for the first possibility (Lefevre et al., 1989).

In order to define more precisely how long heterologous insertions recombine, we have carried out a study using a set of chromosomal insertions of different sizes, located at the same site on the pneumococcal chromosome. We must point out that the formation of donor/recipient heteroduplexes generated by either donor deletions or by donor insertions should differ in more aspects than just the symmetry of donor/recipient heteroduplexes. During DNA entry, natural breaks will occur within an insertion, generating a loss of flanking homology, while deletions escape entry nicks just as point mutations. In addition, pairing of donor fragments containing insertions certainly requires the single-stranded heterologous insertion for folding, while recombination of deleted fragments should involve the bypass of the double-stranded chromosomal region. Both processes are likely to be different, and it was not directly possible to extrapolate previous results obtained with deletions to the behaviour of insertions in genetic crosses. This work was therefore carried out in order to clarify this.

\section{METHODS}

Strains, media and transformation procedures. Streptococcus pneumoniae strains used and constructed in this study derive from strain R36A of Avery (Avery et al., 1944). They have a R801 genetic background (Lefevre $e t$ al., 1979) i.e. wild-type except for a mutation in the bex $B$ gene. Thus they transform all point mutations with the same high efficiency (Tiraby \& Sicard, 1973a). The str-41 marker, conferring resistance to streptomycin, was present in strain R119 (Tiraby \& Fox, 1973). pol $A$ mutations were characterized in $S$. pneumoniae (Martinez et al., 1986) and transferred into the R 801 background as described by Pasta \& Sicard (1994). Culture media, preparation of chromosomal DNA and pneumococcal transformation methods were as described by Claverys et al. (1980). Routinely for a transformation involving $1 \mathrm{ml}$ diluted competent cells, we used about $0 \cdot 2 \mu \mathrm{g}$ chromosome and $0 \cdot 1 \mu \mathrm{g}$ plasmid, i.e. saturating

amounts of DNA. Plasmid- and M13-derived recombinant vectors were amplified in the Eschericbia coli strain JM101 [ $\Delta$ (lac-pro)tbi supE $\mathrm{F}^{\prime}$ traD36 pro $A B$ lacI $\left.I^{\circ} Z \Delta \mathrm{M} 15\right]$ (Messing et al., 1981). Double-stranded and single-stranded DNAs were prepared as described by Maniatis et al. (1982).

Pneumococcal crosses involving the ami locus. The ami locus is an operon involved in oligopeptide transport (Alloing et al., 1990). ami strains are resistant to $0.02 \mathrm{mM}$ methotrexate, but their growth is inhibited on synthetic medium containing excess isoleucine (Sicard, 1964). Conversely, $\mathrm{ami}^{+}$bacteria are sensitive to methotrexate, but they grow on synthetic medium containing excess isoleucine. One-point crosses can therefore be carried out in both directions: transformation of an $a m i^{+}$recipient with ami DNA or vice versa. The transformation efficiency of the donor site is then measured as the number of ami or $\mathrm{ami}^{+}$transformant colonies divided by the number of transformants for the reference point mutation str-41, also carried on the donor DNA and conferring resistance to streptomycin. The possible screening of $\mathrm{ami}^{+}$bacteria allows one to perform two-point crosses. In such crosses, a strain mutated within the ami locus is transformed with another ami mutation, together with the reference marker str-41. $a m i^{+}$bacteria, generated by recombination between the two sites involved in the cross, are selected on synthetic medium. Their number is divided by the number of $\mathrm{Str}^{\mathrm{r}}$ transformants and multiplied by 100 to give the recombination index. In bex backgrounds, the recombination index obtained with point ami mutations depends mainly on the distance separating the two sites. On average, $27 \mathrm{bp}$ lead to a recombination index of $1 \%$ (Claverys et al., 1979).

Insertion of heterologous insertions at the BamHI site of the ami locus. The EcoRI A fragment of the ami locus is approximately $1800 \mathrm{bp}$ in size, with a BamHI site at approximately $1100 \mathrm{bp}$ from the EcoRI right end (Fig. 1). The recombinant plasmid $\mathrm{pR} 6$ carries almost all of the wild-type ami locus on a $5800 \mathrm{bp}$ insert (Méjean et al., 1981). We have subcloned the wild-type allele of the A fragment from $\mathrm{pR} 6$ into the EcoRI site of a M13mp11 replicative form (RF), from which we previously deleted the SmaI-HincII fragment, containing the only BamHI site in M13mp11 (Messing \& Vieira, 1982). The resulting recombinant vector, $\mathrm{RV} 15$, carries the wild-type $A$ fragment with the unique BamHI site. RV15 was linearized with $B a m \mathrm{HI}$ to insert heterologous insertions. A small insertion of 4 bp was introduced by filling in the recessed BamHI ends with 'Klenow fragment' (Maniatis et al., 1982) and ligating with T4 DNA ligase. Among clones resistant to Bam HI digestion, two were sequenced (Sanger et al., 1977); both carry the 4 bp insertion at the Bam HI site. Longer insertions correspond to Sau3A fragments of the pBR322 plasmid (Rodriguez et al., 1977). A Sau3A digest of pBR322 generates 22 fragments from 8 to $1374 \mathrm{bp}$. The two longer ones, 1374 and $665 \mathrm{bp}$, well separated on an agarose gel, were directly purified and ligated to RV15, linearized with BamHI and 5'-dephosphorylated (Maniatis et al., 1982). Smaller Sau3A fragments from pBR322 are located within a smear. To define more precisely the Sau3A sequences to be cloned, we first generated prefragments that separate well on a gel by hydrolysing pBR322 with enzymes that cut rarely. Two such prefragments were purified. One is AseI-HincII, of $368 \mathrm{bp}$. It contains three Sau3A sites generating four fragments, including $18 \mathrm{bp}$ and $46 \mathrm{bp}$ Sau3A sequences that can be ligated to BamHI termini. The second prefragment is BamHI-NruI, of $598 \mathrm{bp}$. When hydrolysed with Sau3A it generates 92 and $359 \mathrm{bp}$ fragments that can be cloned into BamHI. Sau3A digests of prefragments were phenol-extracted, ethanol-precipitated and ligated to RV15, hydrolysed with $B a m \mathrm{HI}$ and dephosphorylated. All ligation mixtures were used to transform separately the $E$. coli strain JM101. RF and viral DNAs were prepared from individual plaques. Recombinant RV15-pBR322 vectors were checked by sequencing and comparing the inserted fragment to the expected sequence of pBR322 (from the EMBL database). We have isolated clones containing the expected sequences of $1374,665,359$ and $92 \mathrm{bp}$. Three clones, generated by ligating the Sau3A digest of the AseI-HincII prefragment into RV15, were sequenced. None carried the $18 \mathrm{bp}$ or the $46 \mathrm{bp}$ sequence, but they carried the complete $64 \mathrm{bp}$ sequence instead. We kept six recombinant clones, each carrying a defined insertion in the Bam HI site of the ami locus (of 4, 64, 92, 359, 665 and $1374 \mathrm{bp}$ ). 


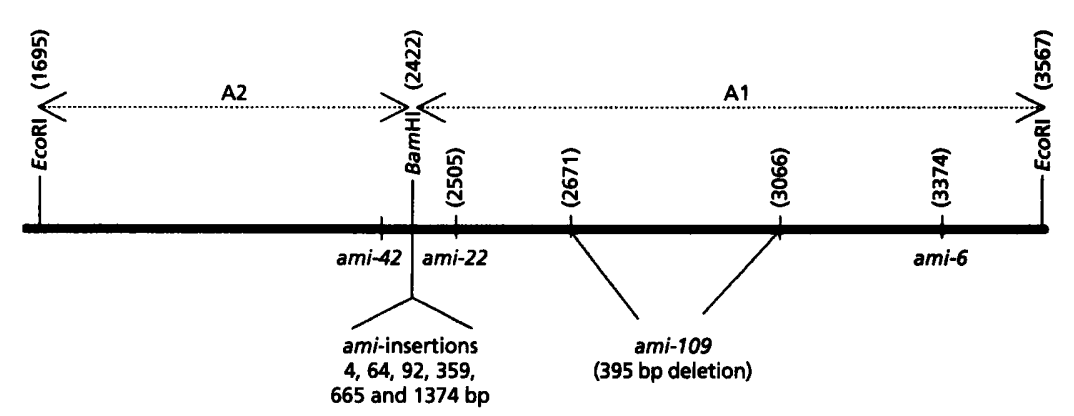

Fig. 1. Physical and genetic map of the $A$ fragment. The ECORI A fragment of the ami locus is about $1800 \mathrm{bp}$. The unique BamHI site divides the $A$ fragment into two subfragments: A2 (EcoRI-BamHI, about $700 \mathrm{bp}$ ) and $A 1$ (BamHI-EcoRI, about $1100 \mathrm{bp})$. The complete wild-type locus and most mutations of the $A 1$ fragment have been sequenced (Alloing et al., 1990; Gasc et al., 1989). ami-6, ami-22 (point mutations) and ami-109 (395 bp deletion) are mapped precisely; positions are in parentheses. Genetic analysis showed that ami-42 is a point mutation located between 30 and 50 bases to the left of the BamHI site on the A2 fragment.

Isolation of pneumococcal strains carrying the ami insertions. RF DNA corresponding to a recombinant clone for a given size of insert was used to transform strain R801. Methotrexateresistant transformants were selected on plates. Such transformants correspond mainly to the desired allelic replacement of the recipient $\mathrm{ami}^{+}$locus by the donor ami-inserted DNA. However, 'insertion-duplication' events integrating the full vector into the chromosome (Vasseghi et al., 1981) also lead to methotrexate resistance, by disrupting the ami locus. The first class of mutations almost never reverts to $\mathrm{ami}^{+}$, while the second class should easily revert by homologous recombination excising the plasmid. For each size of insertion, three ami transformant colonies were picked, subcultured three times in rich medium and plated on synthetic medium to score for $\mathrm{ami}^{+}$ revertants. Out of 18 ami transformants, 2 gave $a m i^{+}$colonies. For each of the six ami-insertion constructs, one nonreverting mutant was kept for further analysis. One set of similar mutants carrying the streptomycin-resistance gene was also constructed by transformation.

Construction of double ami mutant strains. Five double ami mutants have been constructed for the purpose of this study: ami-42 ami-6; ami-ins-1374 ami-6; ami-ins-665 ami-6; ami-ins-359 ami-6; ami-42 ami-109 (see Fig. 1 for mutations). Double mutants were constructed by transformation of a defined mutant with a second mutation. To increase transformation frequency at a genetic site, we used cloned ami mutations as donor DNAs. The point mutant ami-42 and insertion mutants ami-ins-1374, ami-ins- 665 and $a m i$-ins- 359 were transformed with the recombinant plasmid pR32, which carries the ami-6 point mutation on a 5800 bp pneumococcal fragment (Méjean et al., 1981), to yield, respectively, the first four mutants indicated above. The recombinant vector RF824 (from A. M. Gasc, this laboratory) is derived from M13mp11. It carries the ami-109 deletion on the A1 fragment (Fig. 1). We have transformed ami-42 with this plasmid to construct the last double mutant indicated above. Double ami mutants are not more resistant to methotrexate than single ones and therefore must be screened by back-crosses. After each transformation, 50-100 colonies were picked on methotrexate plates and cultured in rich medium. Chromosomal DNA was extracted and used to transform both parental strains. $\mathrm{ami}^{+}$transformants were scored by plating on synthetic medium. Only double mutants cannot produce any wild-type recombinants in both crosses. Among strains giving no $\mathrm{ami}^{+}$ transformants, i.e. 5-25\% of the picked colonies, one from each class was selected for further analysis. Double-mutant genotypes were checked by transformation with the two appropriate parental DNAs and by observing that no $a m i^{+}$transformants were produced.

\section{RESULTS}

\section{Transformation efficiencies of insertions}

Chromosomal DNA from the six strains containing a heterologous insertion within the ami locus was used to transform R801, which is a bex strain. This study is therefore free of any interference from Hex, the generalized mismatch repair system of $S$. pneumoniae.

In agreement with previous findings (Gasc et al., 1987), the smallest insertion of 4 bp transforms as efficiently as a point mutation, since its transformation efficiency is approximately 1 (Table 1 ). With respect to this reference value, we observe that longer insertions have a reduced transformation efficiency. This is particularly noteworthy for the longest insertion, which displays an efficiency of 0.55 (Table 1). Insertions therefore exhibit a decreased efficiency of transformation, inversely related to their size.

To understand more completely the efficiencies of transformation, we performed the reciprocal crosses. The six strains containing an insertion were used as recipients. Donor DNA was $a i^{+}$and Str ${ }^{r}$. We must point out that this situation, i.e. wild-type locus on the donor and insertion on the recipient, is physically identical to crosses with deletions on the donor. Thus, these reciprocal crosses allow the measurement of the transformability of a set of donor deletions of increasing size, flanked by the same DNA sequences. We still observe a decrease in the efficiency, ranging from 1 for the smallest insertions down to 0.36 for the longest ones. Comparing these efficiencies to those with donor insertions, it appears that deletions transform slightly less efficiently than donor insertions.

\section{Recombination of insertions in two-point crosses}

In the two-point crosses between linked ami mutations, one was located on the donor DNA, the other on the recipient chromosome. The recombination index depends on breaks and recombination events occurring between the two ami sites and is therefore mostly proportional to the physical distance separating them. On average, for point mutations in a bex background, a recombination index of $1 \%$ corresponds to a distance of $27 \mathrm{bp}$ between 


\section{Table 1. Effect of insertion length on transformation efficiency}

Efficiency is measured as the ratio of either ami to $\operatorname{Str}^{r}$ transformants (donor insertions, wild-type recipient; central column), or $a i^{+}{ }^{+}$to $\mathrm{Str}^{r}$ transformants (wild-type donor, recipient insertions; right column). Each value is the mean efficiency measured from three independent transformations with two platings, each time screening for ami or $a m i^{+}$and $\operatorname{Str}^{\mathrm{r}}$ colonies.

\begin{tabular}{|ccc|}
\hline Insertion length (bp) & \multicolumn{2}{c|}{ Transformation efficiency } \\
\cline { 2 - 3 } & $a m i$-ins donor $\times \boldsymbol{a m i}^{+}$recipient & $\boldsymbol{a m i}^{+}$donor $\times a m i$-ins recipient \\
\hline 1374 & 0.55 & 0.37 \\
665 & 0.63 & 0.36 \\
359 & 0.64 & 0.51 \\
92 & 0.78 & 0.80 \\
64 & 0.80 & 1.03 \\
4 & 1.03 & 0.98 \\
\hline
\end{tabular}

Table 2. Effect of insertion length on recombination index in two-point crosses involving donor insertions

Chromosomal DNAs carrying both an ami insertion and the Str ${ }^{r}$ marker were used to transform either the ami-22 or the ami-42 mutant. $a m i^{+}$recombinants and $\operatorname{Str}^{\mathrm{r}}$ transformants were counted from three platings and the mean values are indicated below.

\begin{tabular}{|ccc|}
\hline Insertion length (bp) & \multicolumn{2}{c|}{ Mean recombination index observed } \\
\cline { 2 - 3 } & ami-22 recipient & ami-42 recipient \\
\hline 1374 & 22 & 13.5 \\
665 & $23 \cdot 4$ & $16 \cdot 2$ \\
359 & 16 & $8 \cdot 6$ \\
92 & $7 \cdot 3$ & $3 \cdot 4$ \\
64 & $8 \cdot 8$ & $4 \cdot 9$ \\
4 & $4 \cdot 8$ & $1 \cdot 2$ \\
\hline
\end{tabular}

the mutations (Claverys et al., 1979). One set of crosses was carried out with insertions located on the donor DNA. The recipient mutation was either ami-42 or ami22. These two point mutations are located, respectively, on the left and on the right of the insertion site (Bam HI) and very close to it (Fig. 1). Crosses involving the $4 \mathrm{bp}$ insertion gave the recombination indices expected for a point mutation, based on the locations of ami-22 and ami42 relative to the insertion site, i.e. 4.8 and $1.2 \%$, respectively (Table 2 ). When using longer insertions, experimental indices of $\mathrm{ami}^{+}$recombinants exceed these reference values, i.e. insertions trigger hyper-recombination. This behaviour amplifies with the length of the heterologous insertion and appears to reach a plateau for the two longest insertions (Table 2). We also observed that hyper-recombination was higher when crosses were performed with ami-22 than with ami-42. For instance, the $665 \mathrm{bp}$ insert generates about $19 \% \mathrm{ami}^{+}$transformants above the $4.8 \%$ expected with ami-22, but $15 \%$ above the $1.2 \%$ expected with ami-42. This differential hyperrecombination between the proximal and the distal recipient marker remains for each insertion (Table 2) and was previously observed in crosses involving long donor deletions (Lefevre et al., 1989). Whatever the mechanism responsible for hyper-recombination, the close vicinity of a long heterologous insertion apparently affects recombination at a genetic site. Similar crosses were also performed for each donor insertion in an $\mathrm{R} 801 \operatorname{pol} A$ background with either ami-22 or ami-42. The lack of DNA polymerase I had no effect on hyper-recombination (data not shown).

A second set of crosses was performed, using insertions in the recipient. Donor DNA was ami-22 str-41 or ami-42 str41 (Table 3). Crosses involving the $4 \mathrm{bp}$ insertion generate numbers of $\mathrm{ami}^{+}$colonies very close to those obtained above, still in agreement with the distances between genetic sites. For longer insertions, we no longer observe hyper-recombination. The numbers of $\mathrm{ami}^{+}$transformants are even below the reference value obtained with the $4 \mathrm{bp}$ insertion, decreasing slightly as the length of the heterologous insertion increases. This decrease mostly disappears when dividing the recombination index for each insert by the corresponding efficiency obtained in crosses 
Table 3. Effect of insertion length on recombination index in two-point crosses involving recipient insertions

Chromosomal DNA carrying the point mutations ami-22 or ami-42, together with the $\operatorname{Str}^{\mathrm{r}}$ marker, was used to transform ami-insertion recipient strains. ami $i^{+}$recombinants and $\mathrm{Str}^{\mathrm{r}}$ transformants were counted on three platings and the mean values are indicated below.

\begin{tabular}{|ccc|}
\hline Insertion length (bp) & \multicolumn{2}{c|}{ Mean recombination index observed } \\
\cline { 2 - 3 } & ami-22 donor & ami-42 donor \\
\hline 1374 & 2 & 0.8 \\
665 & 2 & 0.5 \\
359 & $2 \cdot 6$ & 0.34 \\
92 & 3.5 & 0.94 \\
64 & 4 & 0.84 \\
4 & $5 \cdot 8$ & $2 \cdot 3$ \\
\hline
\end{tabular}

(a)

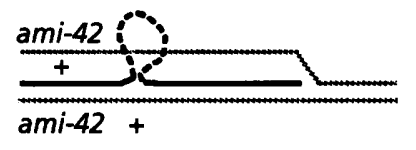

(b)

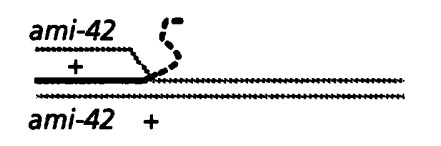

(c)

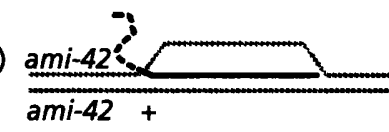

(d)

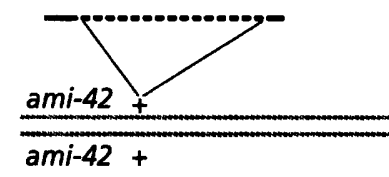

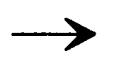
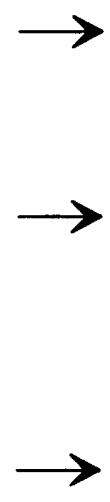
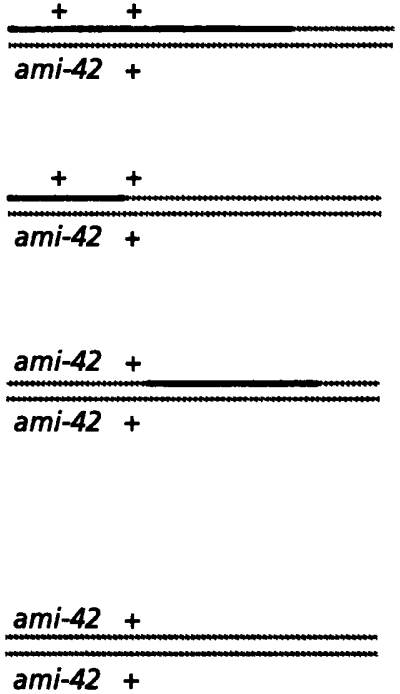

Fig. 2. Models accounting for the reduced transformation efficiency and hyperrecombination of insertions. (a) Pairing is completed successfully, generating a singlestranded loop which may be repaired to wild-type. (b, c) Exclusion or one-sided pairings: recombination stops because heterology is a strong barrier and/or because homology on one side is absent or very short. (d) Both flanking homologies are too short; there is no recombination at all. Situations (a), (b), (c) and (d) account for the reduced efficiency of insertions. Situations (a) and (b) also explain hyper-recombination in the two-point cross symbolized in this figure. Black line, donor strand; grey lines, recipient strands; dashed line, heterologous ami-insertion. between donor $a m i^{+}$and recipient $a m i$-insertions (calculation not shown). Thus, the reduced efficiencies and the slight hyporecombination observed when using recipient insertions are likely to have the same cause.

\section{Testing for a loop repair or one-sided pairings}

The results described above show that, with respect to point mutations, long donor insertions have a reduced transformation efficiency in one-point crosses and induce hyper-recombination in two-point crosses. We propose two models to account for this behaviour (Fig. 2). The same models may also account for hyporecombination due to recipient insertions.

First, recombination of a fragment carrying an insertion should generate a single-stranded heteroduplex loop. Such structures might undergo correction biased to the wild-type sequence and be closely localized around the loop to prevent co-conversion, as shown in Fig. 2(a). In addition, this correction would not be dependent on DNA polymerase I, since pol $A$ mutants allow hyperrecombination to occur. The second model, the exclusion model, is based on the fact that a long insert is a barrier which might inhibit recombination of donor DNA. Moreover, donor fragments broken within the insertion cannot fully recombine. Such one-sided pairings, shown in Fig. 2(b, c), will exclude the long insertion, broken or not, from the pairing synapsis, thus reducing their efficiency. These events will also produce hyper-recombination (Fig. 2b).

In order to distinguish between the two possibilities, we have carried out two kinds of three-point crosses. In a first set of crosses (three-point cross of type I, Fig. 3), the double mutant strain ami-42 ami-6 was used as recipient. ami-42 and ami-6 flank the insertion site BamHI (Fig. 1). ami-insertions were located on the donor DNA. In this situation a loop repair event should generate a wild-type transformant when the central insertion and both flanking 
(a)


(b)



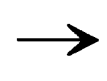

Fig. 3. Three-point cross of type I. This class of cross was used to test the loop repair model in a positive way. ami-insertions were used to transform the ami-42 ami-6 double mutant. A localized loop-repair event (a) should mostly generate a wild-type transformant, as in the two-point cross of Fig. 2(a). One-sided pairings (b), which exclude the heterologous insertion and flanking DNA, should lead to a mutant genotype, while they generate an $\mathrm{ami}^{+}$ genotype in the two-point crosses of Fig. 2(b). Line symbols are as in Fig. 2.

Table 4. Effect of insertion length on recombination indices in three-point crosses of type 1

The double mutant ami-42 ami-6 was transformed with the ami-insertions, as shown in Fig. 3.

\begin{tabular}{|cccc|}
\hline Insertion length (bp) & \multicolumn{3}{c|}{ Mean recombination index values } \\
\cline { 2 - 4 } & Observed* & $\begin{array}{c}\text { Predicted by a loop } \\
\text { repair model } \dagger\end{array}$ & $\begin{array}{c}\text { Predicted by double } \\
\text { recombinations } \ddagger\end{array}$ \\
\hline 1374 & 0.68 & 10.5 & 0.4 \\
665 & 0.62 & 12.5 & 0.4 \\
359 & 0.53 & 6.7 & 0.4 \\
92 & 0.37 & 2.7 & 0.4 \\
64 & 0.19 & 3.7 & 0.4 \\
4 & 0.38 & - & 0.4 \\
\hline
\end{tabular}

* Observed indices were calculated as described in Methods.

$\dagger$ These are the expected indices if repair is the cause of hyper-recombination. With respect to the situation of Fig. 2(a), the number of loop repairs leading to a wild-type strand should be reduced by about $23 \%$, i.e. the frequency of events integrating $a m i-42^{+}$and $a m i$-ins, without integrating $a m i-\sigma^{+}$. Values observed in two-point crosses (Table 2, ami-42 recipient) were multiplied by $77 \%$ to give the predicted indices. This calculation is not relevant for the control insertion of 4 bp which does not induce hyper-recombination.

$\ddagger$ Values correspond to independent recombination events: integration of ami-42 ${ }^{+}$without ami-ins (about $1 \cdot 8 \%$ ) and integration of $a m i-\sigma^{+}$without ami-ins (about $23 \%$ ).

wild-type sites cointegrate into the chromosome, as in Fig. 3(a). Thus, if the hyper-recombination observed in crosses between the ami-42 recipient and the long amiinsertion donors is due to loop correction, it will be mostly maintained in the present cross, as ami- 6 is linked to the insertion site. Predictive calculations are in Table 4. Conversely, if hyper-recombination results mainly from one-sided pairings, the number of wild-type transformants should be lowered considerably, as a point mutation still remains on the chromosome (Fig. 3b). Experimental values are very close to the number of wildtype transformants predicted by double recombination events (Table 4), i.e. independent integrations of ami-42+ and of ami- $\sigma^{+}$without integration of the central heterologous insertion, as explained in the legend of Table 4. The results of these crosses do not support a loop repair model. On the other hand, they are compatible with a onesided pairing model, although they do not directly prove it.
To obtain evidence directly supporting the latter possibility, i.e. the exclusion model, also called a one-sided pairing model, we have carried out a second kind of cross (three-point cross of type II, Fig. 4). Three double ami mutant strains were constructed, containing one of the larger ami-insertions and the ami-6 mutation. The streptomycin-resistance marker was also present in these strains. Chromosomal DNA from these mutants was used to transform the $a m i-42$ recipient strain. The following is assumed: with respect to crosses between the ami-42 recipient and the ami-insertion donors, the exceedingly large number of $\mathrm{ami}^{+}$bacteria should be mostly unchanged in such three-point crosses provided that onesided pairings are responsible for hyper-recombination. Indeed, according to this model the ami- 6 mutation which flanks the heterologous insertion should be excluded with it, or unlinked by breaks occurring within the insertion (Fig. 4a). In addition, one should note that in this kind of cross, loop repair events cannot generate a wild-type 
(a)

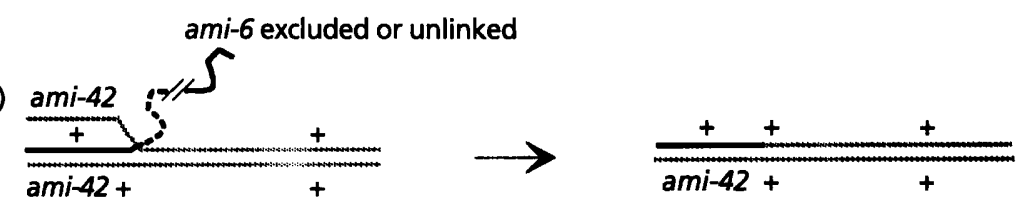

(b)



Fig. 4. Three-point cross of type II. This class of cross was used to test the exclusion model in a positive way. (a) The insertion being excluded (the linked point mutation ami-6) should also be excluded from the pairing or should be unlinked by entry breaks within the insertion. This should lead to a wild-type recombinant as in the twopoint cross of Fig. 2(b). (b) Loop corrections should not frequently lead to a wild-type transformant, with respect to the two-point cross of Fig. 2(a). Line symbols are as in Fig. 2.

\section{Table 5. Effect of insertion length on recombination indices in three-point crosses of type II}

The ami-42 mutant was transformed with the doubly mutated DNA (ami-ins- 1374 ami-6, ami-ins- 665 ami-6 or ami-ins-359 ami-G) as shown in Fig. 4.

\begin{tabular}{|lccc|}
\hline Insertion length (bp) & \multicolumn{3}{c|}{ Mean recombination index values } \\
\cline { 2 - 4 } & Observed* & Predicted (exclusion) $\dagger$ & Predicted (correction) $\ddagger$ \\
\hline 1374 & 16 & $13 \cdot 5$ & $3 \cdot 1$ \\
665 & 14 & $16 \cdot 2$ & $3 \cdot 7$ \\
359 & $7 \cdot 6$ & $8 \cdot 6$ & 2 \\
\hline
\end{tabular}

* Observed indices are calculated as described in Methods.

† Expected values if exclusion is the cause of hyper-recombination. The prediction is as follows. As ami6 is excluded with the heterologous insertion (Fig. 4a), the situation is identical to that of Fig. 2(b) and should lead to the same indices. Thus values predicted from the exclusion model are those from Table 2 (ami-42 recipient).

$\ddagger$ Predicted $a m i^{+}$indices, based on a loop correction model. The prediction is as follows. The situation becomes identical to Fig. 2(a) when ami-ins integrates without ami-6. As the disjunction frequency between ami- 6 and the insertion site is about $23 \%$, ami $i^{+}$numbers should be reduced to $23 \%$ of those observed in two-point crosses (Table 2, ami-42 recipient), thus giving the numbers indicated.

genotype (Fig. 4b), unless a recombination occurs between ami-6 and the right border of the insertion. Therefore, based on a multibase loop repair model, the number of $\mathrm{ami}^{+}$transformants observed in crosses between the ami-42 recipient and the ami-insertion donors should be drastically affected by the presence of the flanking ami-6 mutation on the donor DNA. Experimental results and expected values according to one model or to the other are detailed in Table 5. We found that the numbers of $\mathrm{ami}^{+}$transformants are identical to those obtained in a cross between the ami-insertion donors and the ami-42 recipient. This result directly supports the exclusion model and, moreover, it does not substantiate a loop correction model.

\section{Using the ami-109 deletion in three-point crosses}

The results with donor insertions indicate that reduced efficiency and hyper-recombination are not consistent with a loop correction model. Reduced transforming efficiencies and hyper-recombination were also characteristic of donor deletions. In particular, results obtained with the 395-base deletion ami-109 (Lefevre et al., 1989) indicated about $20 \%$ hyper-recombination. However, this excess of $\mathrm{ami}^{+}$recombinants was attributed to a loop correction pathway, not to an exclusion of the donor deletion. To determine whether completely different mechanisms for donor insertion and donor deletion account for similar results, i.e. reduced efficiency and hyper-recombination, we have reinvestigated the behaviour of a donor deletion. For this purpose we carried out the same three-point crosses as described above, but involving ami-109 as a long heterologous insertion.

First, the double mutant strain ami-42 ami-6 was transformed with donor DNA carrying ami-109 (Fig. 5). This deletion is located approximately in the middle of the two sites (Fig. 1). A conversion of the deletion to the wild-type sequence suggests that hyper-recombination remains (Fig. $5 a)$. Actually, the situation is identical to a cross between the ami-109 deletion donor and the ami-6 recipient, 
(a)
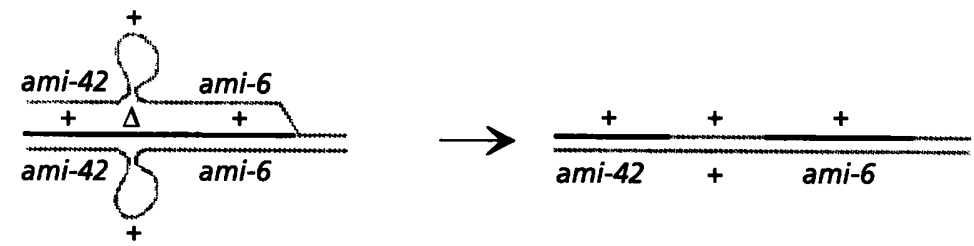

(b)

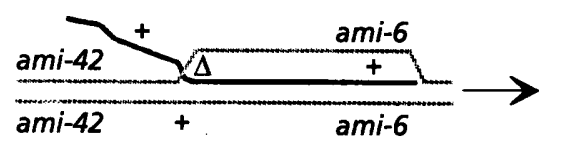

$\frac{a m i-42++\frac{+}{a m i-6}}{a m i-42+}$

Fig. 5. Testing the ami-109 deletion in a three-point cross of type 1 . Conversion of the deletion to wild-type leads to an $\mathrm{ami}^{+}$ strand (a). Exclusion of the donor strand at the heterologous insertion allows one flanking mutation to be maintained on the chromosome (b). $\Delta$, ami-109 deletion. Line symbols are as in Fig. 2. (a)
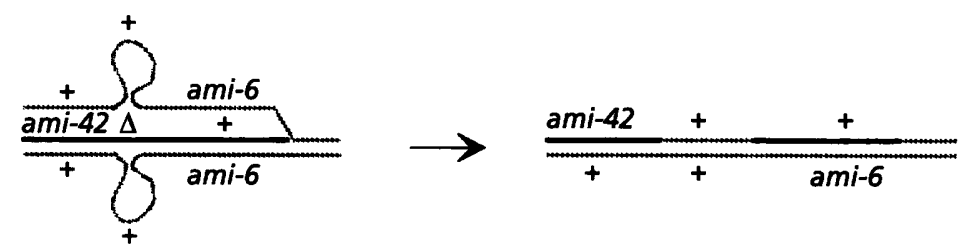

(b)

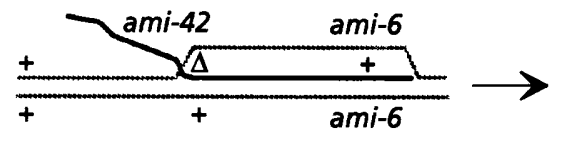

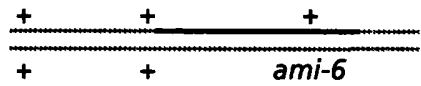

Fig. 6. Testing the ami-109 deletion in a three-point cross of type II. (a) A correction changing the deletion to wild-type has no effect on the ami-42 donor mutation; the strain remains mutant. (b) Pairing stops at the deletion, thus preventing the ami-42 mutation from being integrated into the recipient chromosome. Symbols are as in Fig. 5. provided that the ami-109 deleted site co-recombines with the ami-42+ site. According to the positions of ami-42 and ami-109, disjunction between the two sites should be about $10 \%$. Consequently, according to a loop correction model, the $\mathrm{ami}^{+}$index generated in the present three-point cross should be reduced by $10 \%$ as compared with crosses between the ami-109 donor and the ami- 6 recipient. Since this latter cross produces around $27 \% \mathrm{ami}^{+}$colonies, the expected value based on a correction model should be approximately $24 \% \mathrm{ami}^{+}$colonies. On the other hand, if one-sided pairings are responsible for the excess of $a m i^{+}$ transformants, this excess should disappear in this cross since one lateral mutation remains on the chromosome (Fig. 5b). We have obtained $0.71 \% \mathrm{ami}^{+}$transformants, i.e. close to the expected number based on independent recombinations integrating both ami-42+ and ami- $\sigma^{+}$sites without integrating the central deletion ami-109. Such events may be estimated at around $1 \%$, since the distance between ami-109 and ami-6 or ami-42 is about $10 \%$.

In addition, we have constructed a double mutant ami-109 ami-42. Its DNA was used to transform the ami-6 mutant strain (Fig. 6). Complete pairing and conversion of the deletion to the wild-type (Fig. 6a) should lead to a drastically reduced number of $\mathrm{ami}^{+}$colonies relative to the cross between the ami-109 donor and the ami- 6 recipient. Since recombination between the ami-109 site and the ami42 site is approximately $10 \%$, the $\mathrm{ami}^{+}$index in the present three-point cross should fall between 2 and $3 \%$. Conversely, if pairing stops at the heterologous insertion, the ami-42 mutation is excluded (Fig. 6b), and the number of $\mathrm{ami}^{+}$colonies should be mostly the same as in a cross between the ami-109 DNA and the ami-6 recipient. We have observed an $\mathrm{ami}^{+}$frequency identical to the one obtained in the cross between the ami-109 donor and the ami- 6 recipient, i.e. between $25 \%$ and $30 \%$. These results firmly support the prediction based on exclusion events to account for recombination of donor deletions. The conversion model, previously proposed (Lefevre et al., 1989), is not confirmed by the present study; we have no satisfactory hypothesis to explain previous observations.

\section{DISCUSSION}

Several insertions and one deletion were used to elucidate the recombination behaviour of long heterologous insertions. By a combination of two- and three-point crosses, we have found that both donor insertions and donor deletions are not repaired at the heteroduplex level. Reduced transforming efficiency and hyperrecombination, induced by long deletions and insertions, are best explained by pairing inhibition excluding the donor heterologous insertion from the heteroduplex joint. Such exclusion may also account for the low efficiency and hyporecombination observed in crosses involving recipient insertions (Table 1, right column, Table 3). In that situation, recombination is identical to transformation involving donor deletions, i.e. the wild-type site should be frequently excluded from the pairing synapsis. In twopoint crosses, while exclusion of donor insertions directly generates a wild-type transformant, exclusion of donor wild-type sites does not. Moreover, such exclusion events are 'lost' for recombining the wild-type site into the chromosome, thus explaining the slight hyporecombina- 
tion observed in two-point crosses involving recipient insertions (Table 3).

Thus, effective pairing of homologous sequences would be initiated by the invading single-strand donor DNA, and the pairing process would be partly blocked at the level of long heterologous insertions. Inhibition is only partial, since the transformation efficiency of long heterologous insertions is reduced approximately to one-third or one-half of the efficiency of point mutations. This is consistent with in vitro studies showing that although RecA of E. coli is able to catalyse recombination of long heterologous insertions, the process is less efficient than for homologous DNA (Bianchi \& Radding, 1983). In addition, regarding pneumococcal transformation, donor insertions must undergo nicks during entry. As the mean size for entering fragments is about 6000 bases (Morrison \& Guild, 1972), insertions of a few hundred bases should be broken with frequencies close to $10 \%$ and more if longer. Such broken insertions cannot be efficient in onepoint crosses whatever the situation, i.e. no recombination at all or recombination on one side only (Fig. 2). Natural breaks must explain, in the case of donor insertions, a significant part of the observed reduction of efficiency and hyper-recombination.

In this study we also found that insertions transform as well as or more efficiently than deletions of corresponding size. This observation was unexpected, because deletions have no physical size and should behave as single point mutations with regard to entry, while insertions undergo breaks. However, this observation is consistent with previous in vitro findings of Bianchi \& Radding (1983): heteroduplexes are spanned more efficiently by RecA when the heterologous insertion is on the single-stranded partner rather than on the duplex DNA. Thus, we propose that the unwinding of a heterologous chromosomal region is the limiting step for the transformation of a donor deletion. It should be a more limiting step than single-stranded DNA folding is for the transformation of a donor insertion. Indeed, in spite of entry breaks destroying them, donor insertions appear to be more transformable markers than donor deletions, at least for the heterologous insertion sizes studied in this work. Lacks (1966) found that the transformation efficiency of the multisite mutation malE9, probably a very long deletion, is $0 \cdot 4$. Conversely, when transforming a malE9 strain with $\mathrm{mal}^{+} \mathrm{DNA}$, i.e. in a situation identical to a donor insertion, the transformation efficiency of the wildtype locus decreased to $0 \cdot 009$. As the malE9 deletion is very large, i.e. close to the mean size of an entering fragment, almost all mal ${ }^{+}$entering fragments should be broken within the sequence deleted on the chromosome, thus explaining the very low efficiency. In our study, insertions are small compared to the mean length of entering DNAs. Interestingly, when carried on donor DNA, the malE9 deletion had a transformation efficiency of 0.4 (Lacks, 1966). This value is very close to those observed in the present study when transforming strains containing the 665 or the 1374 bp insertions with $a m i^{+}$ DNA. This observation could indicate that, although a multibase chromosomal heterologous insertion partly inhibits recombination of the donor single-stranded fragment, it can be bypassed in vivo, and this bypass could occur efficiently whatever the heterologous insertion size. This initial decrease of recombination for DNAs carrying heterologous insertions beyond a few hundred bases, followed by a plateau for longer heterologous insertions, is reminiscent of the findings of $\mathrm{Bi} \& \mathrm{Liu}$ (1994). They characterized two kinds of recombination in $E$. coli: $\operatorname{rec} A$ independent recombination, sensitive to the heterologous insertion length between two repeats, and $\operatorname{rec} A$-dependent recombination, sensitive to homology sizes.

The crosses carried out in this study suggest that no pathway specialized for correcting multibase loop heteroduplexes exists in $S$. pneumoniae. Nevertheless, such structures must be formed when recombination of a long donor heterologous insertion is completed successfully; this is likely to occur as long heterologous insertions are transformable markers. Thus, $S$. pneumoniae appears not to correct big looped-out heteroduplexes, while most point mismatches are repaired; regarding the respective bulks, this may appear paradoxical. However, it is likely that this situation is satisfactory for bacteria. Similarly one should recall, for example, that $\mathrm{C} / \mathrm{C}$ mismatches appear to be very destabilizing for the DNA structure (Gasc et al., 1989). Nevertheless $C / C$ escapes correction by the Hex pathway (Claverys et al., 1983) and is poorly repaired by the $E$. coli Mut system (Kramer et al., 1984). Indeed, this mismatch should not be detrimental to bacteria: transversions, especially $G \rightarrow C$, do not arise frequently because of the proofreading activity of DNA polymerase III (Wu et al., 1990). Conversely, A/C and G/T, which are efficiently repaired by the Hex and Mut systems, result from transitions, which are not efficiently repaired by the proofreading activity of DNA polymerase III (Wu et al., 1990). In the same perspective, we can imagine that looped-out heteroduplexes do not arise frequently in bacterial chromosomes and that specific counteracting systems have not evolved in bacteria. In addition, considering natural transformation, pneumococci could benefit by retrieving through recombination, long heterologous or heterospecific DNA fragments. Such horizontal transfers of blocks of foreign DNA have been proposed to explain the emergence of highly penicillin-resistant strains of $S$. pneumoniae among hospital isolates (Dowson et al., 1989). Indeed, we can imagine that like heterologous insertions in this study, highly divergent regions could, sometimes, lead to the formation of heteroduplex bubbles and be integrated into a foreign chromosome, provided there is enough homology on flanking sides.

In $E$. coli, no repair system specializing in long multibase loop heteroduplexes was found (Carraway \& Marinus, 1993). On the other hand, based on tetrad analysis, it was concluded that two long deletions undergo conversion in yeast (Fogel et al., 1981). Correction mechanisms acting on single-strand bubble heteroduplexes have been demonstrated in mammals (Ayares et al., 1987; Weiss \& Wilson, 1987). The large size and high sequence redundancy of eukaryotic genomes may frequently induce polymerase slippage or interfere with meiotic recombinations. Subsequent looped-out structures could arise in high prop- 
ortions and be toxic for cells unless they are repaired by specific pathways.

\section{ACKNOWLEDGEMENTS}

We are indebted to Caroline Monod for linguistic assistance. This work was supported, in part, by the Universite Paul Sabatier.

\section{REFERENCES}

Alloing, G., Trombe, M. C. \& Claverys, J. P. (1990). The ami locus of the Gram-positive bacterium Streptococcus pneumoniae is similar to binding protein-dependent transport operons of Gram-negative bacteria. Mol Microbiol 4, 633-644.

Avery, O. T., MacLeod, C. M. \& MacCarty, M. (1944). Studies on the chemical nature of the substance inducing transformation of pneumococcal types. Induction of transformation by a deoxyribonucleic acid fraction isolated from pneumococcus type III. $J$ Exp Med 89, 157-158.

Ayares, D., Ganea, D., Chekuri, L., Campbell, C. R. \& Kucherlapati, R. (1987). Repair of single-stranded DNA nicks, gaps, and loops in mammalian cells. Mol Cell Biol 7, 1656-1662.

Bi, X. \& Liu, L. F. (1994). $\operatorname{rec} A$-independent and $\operatorname{rec} A$-dependent intramolecular plasmid recombination. Differential homology requirement and distance effect. J Mol Biol 235, 414-423.

Bianchi, M. E. \& Radding, C. M. (1983). Insertions, deletions and mismatches in heteroduplex DNA made by RecA protein. Cell 35, 511-520.

Carraway, M. \& Marinus, M. G. (1993). Repair of heteroduplex DNA molecules with multibase loops in Eschericbia coli. J Bacteriol 175, 3972-3980.

Claverys, J.-P. \& Lacks, S. A. (1986). Heteroduplex deoxyribonucleic acid base mismatch repair in bacteria. Microbiol Rev 50, $133-165$.

Claverys, J.-P., Lataste, H. \& Sicard, A. M. (1979). Localization of two EcoRI restriction sites within the ami $A$ locus in pneumococcus: relationship between the physical and the genetic map. In Transformation 1978, pp. 161-169. Edited by S. W. Glover \& L. O. Butler. Oxford: Cotswold Press.

Claverys, J.-P., Roger, M. \& Sicard, A. M. (1980). Excision and repair of mismatched base pairs. Mol \& Gen Genet 178, 191-201.

Claverys, J.-P., Mejean, V., Gasc, A. M. \& Sicard, A. M. (1983). Mismatch repair in Streptococcus pneumoniae: relationship between base mismatches and transformation efficiencies. Proc Natl Acad Sci US $A$ 80, 5956-5960.

Dowson, C. G., Hutchinson, A., Brannigan, J. A., George, R. C., Hansman, D., Linares, J., Tomasz, A., Maynard-Smith, J. \& Spratt, B. G. (1989). Horizontal transfer of penicillin binding protein genes in penicillin resistant clinical isolates of Streptococcus pneumoniae. Proc Natl Acad Sci US A 86, 8842-8846.

Ephrussi-Taylor, H. \& Gray, T. C. (1966). Genetic studies of recombining DNA in pneumococcal transformation. $J$ Gen Pbysiol 49, 211-231.

Fogel, S., Mortimer, R. K. \& Lusnak, K. (1981). Mechanisms of meiotic gene conversion, or 'wanderings on a foreign strand'. In The Molecular Biology of the Yeast Saccharomyces: Life Cycle and Inberitance, pp. 289-339. Edited by J. N. Strathern, E. W. Jones \& J. R. Broach. Cold Spring Harbor, NY: Cold Spring Harbor Laboratory.

Gasc, A. M., Garcia, P., Baty, D. \& Sicard, A. M. (1987). Mismatch repair during pneumococcal transformation of small deletions produced by site-directed mutagenesis. Mol \& Gen Genet 210, 369-372.

Gasc, A. M., Sicard, A. M. \& Claverys, J.-P. (1989). Repair of single and multiple mismatches during recombination in Streptococcus pneumoniae. Genetics 120, 29-36.

Ghei, O. M. K. \& Lacks, S. A. (1967). Recovery of donor deoxyribonucleic acid marker activity from eclipse in pneumococcal transformation. J Bacteriol 93, 816-829.

Kramer, B., Kramer, W. \& Fritz, H.-J. (1984). Different base/base mismatches are corrected with different efficiencies by the methyldirected DNA mismatch-repair system of E. coli. Cell 38, 879-887.

Lacks, S. (1966). Integration efficiency and genetic recombination in pneumococcal transformation. Genetics 53, 207-235.

Lacks, S. A. (1970). Mutants of Diplococcus pneumoniae that lack deoxyribonucleases and other activities possibly pertinent to genetic transformation. J Bacteriol 101, 373-383.

Lacks, S. (1988). Mechanisms of genetic recombination in Grampositive bacteria. In Genetic Recombination, pp. 43-86. Edited by R. Kucherlapati \& G. R. Smith. Washington, DC: American Society for Microbiology.

Lefèvre, J. C., Claverys, J.-P. \& Sicard, A. M. (1979). Donor deoxyribonucleic acid length and marker effect in pneumococcal transformation. J Bacteriol 138, 80-86.

Lefèvre, J. C., Mostachfi, P., Gasc, A. M., Guillot, E., Pasta, F. \& Sicard, M. (1989). Conversion of deletions during recombination in pneumococcal transformation. Genetics 123, 455-464.

Maniatis, T., Fritsch, E. F. \& Sambrook, J. (1982). Molecular Cloning: a Laboratory Manual. Cold Spring Harbor, NY: Cold Spring Harbor Laboratory.

Martinez, S., Lopez, P., Espinosa, M. \& Lacks, S. A. (1986). Cloning of a gene encoding a DNA polymerase-exonuclease of Streptococcus pneumoniae. Gene 44, 79-88.

Méjean, V. \& Claverys, J.-P. (1984). Effect of mismatched base pairs on the fate of donor DNA in transformation of Streptococcus pneumoniae. Mol \& Gen Genet 197, 467-471.

Méjean, V., Claverys, J.-P., Vasseghi, H. \& Sicard, A. M. (1981). Rapid cloning of specific DNA fragments of Streptococcus pneumoniae by vector integration into the chromosome followed by endonucleolytic excision. Gene 15, 289-293.

Messing, J. \& Vieira, J. (1982). A new pair of M13 vectors for selecting either DNA strand of double digest restriction fragments. Gene 19, 269-276.

Messing, J., Cua, R. \& Seeburg, P. H. (1981). A system for shotgun DNA sequencing. Nucleic Acids Res 9, 309-321.

Morrison, D. A. \& Guild, W. R. (1972). Transformation and deoxyribonucleic acid size: extent of degradation on entry varies with size of donor. $J$ Bacteriol 112, 1157-1168.

Pasta, F. \& Sicard, M. (1994). Hyperrecombination in pneumococcus, $A / G$ to $C . G$ repair and requirement for DNA polymerase I. Mutat Res 315, 113-122.

Rodriguez, R. L., West, R. W., Heyneker, H. L., Bolivar, F. \& Boyer, H.W. (1977). Characterization of tetracycline and ampicillin resistant plasmid cloning vehicles. In Molecular Cloning of Recombinant $D N A$, pp. 73-84. Edited by W. A. Scott \& H. W. Werner. Miami Winter Symposia. New York: Academic Press.

Sanger, F., Nicklen, S. \& Coulson, A. R. (1977). DNA sequencing with chain-terminating inhibitors. Proc Natl Acad Sci USA 74, 5463-5467.

Sicard, A. M. (1964). A new synthetic medium for Diplococcus pneumoniae and its use for the study of reciprocal transformation at the ami locus. Genetics 50, 31-44. 
Tiraby, G. \& Fox, M. S. (1973). Marker discrimination in transformation and mutation of pneumococcus. Proc Natl Acad Sci US A 70, 3541-3545.

Tiraby, G. \& Sicard, A. M. (1973a). Integration efficiency in DNAinduced transformation of pneumococcus. II. Genetic studies of a mutant integrating all the markers with a high efficiency. Genetics 75, 23-33.

Tiraby, G. \& Sicard, A. M. (1973b). Integration efficiencies of spontaneous mutant alleles of ami $A$ locus in pneumococcal transformation. J Bacteriol 116, 1130-1135.

Vasseghi, H., Claverys, J.-P. \& Sicard, A. M. (1981). Mechanism of integrating foreign DNA during transformation of Streptococcus pneumoniae. In Proceedings of the 5th European Meeting on Transformation and Transfection, pp. 137-154. Edited by G. Polsinelli \& G. Mazza. Oxford: Cotswold Press.

Weiss, U. \& Wilson, J. H. (1987). Repair of single-stranded loops in heteroduplex DNA transfected into mammalian cells. Proc Natl Acad Sci US A 84, 1619-1623.

Wu, T.-H., Clarke, C. H. \& Marinus, M. G. (1990). Specificity of Escherichia coli mutD and mutL mutator strains. Gene 87, 1-5.

Received 7 August 1995; revised 3 October 1995; accepted 13 October 1995. 